\title{
Effects of Ascorbic Acid on Iproniazid-Induced Hepatitis in Phenobarbital-Treated Rats
}

\author{
Yōko Matsuki, ${ }^{* a}$ Rie Bandou, ${ }^{a}$ Hiroshi Kiwada, ${ }^{a}$ Harumi Maeda, ${ }^{b}$ and \\ Tsuyoshi GOROMARU ${ }^{b}$ \\ Faculty of Pharmaceutical Sciences, University of Tokushima, ${ }^{a} 78$, Shomachi 1-chome, Tokushima 770, Japan and \\ Faculty of Pharmacy and Pharmaceutical Sciences, Fukuyama University, ${ }^{b} 985$, Sanzou, Higashimura-cho, Fukuyama \\ 729-02, Japan. Received January 5, 1994; accepted April 1, 1994
}

\begin{abstract}
The effects of ascorbic acid (AA) on hepatic injury induced by iproniazid (IPN) in phenobarbital-treated rats were investigated by the evaluation of hepatic function using the clearance of aminopyrine (AM). Either IPN or isopropylhydrazine (IP-Hy), a potent toxic metabolite of IPN, were administered as a pretreatment to rats with or without AA. After i.v. injection of AM, the blood concentration of AM was determined by capillary gas chromatography by isotope dilution analysis using deuterium-labeled AM (AM- $\left.d_{9}\right)$ as the internal standard. The kinetic parameters of $\mathrm{AM}, V_{\mathrm{d}}, \boldsymbol{k}_{\mathrm{el}}$ and total body clearance, were estimated from the time course of blood concentration. Pretreatment with IPN with AA led to a marked increase in the $k_{\mathrm{el}}$ and in the clearance compared with pretreatment using IPN alone. A significant increase in the $k_{\mathrm{el}}$ and the clearance was also found in the case of combined pretreatment using IP-Hy with AA.

The effects of AA on the hepatic injury induced by IPN were studied according to its histological aspects. In the specimens obtained following the administration of IPN or IP-Hy with AA, the degree of cell necrosis was remarkably lowed both quantitatively and qualitatively.

The present results clearly demonstrate that $\mathrm{AA}$ was effective in reducing IPN-induced hepatitis.
\end{abstract}

Keywords iproniazid; isopropylhydrazine; ascorbic acid; aminopyrine clearance; hepatic function; iproniazid-hepatitis

Hydrazine (Hy) derivatives have been widely utilized as antituberculosis agents, the monoamine oxidase (MAO) inhibitors, the anticancer drugs and the antihypertensive agents. However, numerable side effects, such as hepatic injury and carcinogenic activity, have been found. ${ }^{1,2)}$ Our previous investigation concerning the effects of ascorbic acid (AA) on the metabolic fate of isoniazid (INAH) indicated that AA led to an extreme delay in the absorption rate of INAH and a significant decrease in the urinary excretion of Hy, a potent toxic metabolite. ${ }^{3)}$ Further, we demonstrated using the microsomal system that AA can inhibit the generation of free radical intermediates caused by INAH, which have been regarded as a trigger for the development of hepatic injury. ${ }^{4}$ Also, we investigated the effects of AA on the metabolic fate of iproniazid (IPN), a derivative of INAH, and indicated that the absorption process and the metabolism of IPN was not affected by AA, and only the amount of urinary excretion of Hy decreased. ${ }^{5)}$ Further, it was defined that generation of free radical intermediates by to IPN, causing hepatic injury, was significantly inhibited by AA. Thus, it was suggested that AA might be effective in reducing hepatic injury due to IPN. ${ }^{\text {) }}$

IPN was developed as an anti-depressant because of its MAO-inhibiting activity, but the drug is not used at present because IPN has hepatic toxicity. Our previous study indicated that IPN-induced liver damage was due to an isopropyl radical which was generated from IPN and isopropylhydrazine (IP-Hy), ${ }^{5}$ and it has been recognized that IPN toxicity may be valuable under various conditions affecting its metabolism. ${ }^{2)}$

The present study is aimed at elucidating the effects of AA on IPN-induced hepatitis in phenobarbital-treated rats by evaluating of hepatic function. Hepatic function was estimated using the clearance of aminopyrine (AM) as the index, which was demonstrated by our previous study ${ }^{6)}$ to highly reflect hepatic function. In addition, we examined hepatic function from the aspects of glutamic oxaloacetic transaminase (GOT) and glutamic pyruvic transaminase (GPT) activity and liver histology.

\section{MATERIALS AND METHODS}

Materials AM, AA, ascorbic acid sodium salt (AA-Na), phenobarbital sodium salt (PB) and Transaminase C II-Test Wako were purchased from Wako Pure Chemical Ind., Ltd. Deuterium-labeled AM (1-phenyl-2methyl-3-trideutero-methyl-4- $N$-di-trideuteromethyl-pyrazoline-5-one, AM- $d_{9}$ ), IPN and IP-Hy were the same as that used in our previous study. ${ }^{6,7)}$ IP-Hy was prepared as IP-Hy sulfate.

Estimation of the Clearance of AM As described in the previous paper, ${ }^{6)}$ through a canule inserted into the femoral vein, AM dissolved in saline was injected into male Wistar rats $(180-230 \mathrm{~g})$ at a dose of $10 \mathrm{mg} / \mathrm{kg}$, and each $100 \mu \mathrm{l}$ of blood was collected from non-anesthetized rats at certain intervals through a canule inserted into the femoral artery until $90 \mathrm{~min}$ after the injection of AM. One $\mathrm{ml}$ of water was added to the blood samples, which then stood for $30 \mathrm{~min}$. After the addition of $1 \mathrm{~g}$ ammonium sulfate, the blood samples stood for $10 \mathrm{~min}$ more, and then $0.5 \mathrm{ml}$ of ethylacetate containing AM- $d_{9}(0.8 \mu \mathrm{g} / \mathrm{ml})$ as an internal standard was added. The mixture was then shaken for $10 \mathrm{~min}$ and centrifuged at $3000 \mathrm{rpm}$ for 10 $\min$. About $200 \mu \mathrm{l}$ of the upper organic layer was transferred into a microtube and evaporated by a stream of $\mathrm{N}_{2}$ gas. After drying, it was stored at room temperature until the time of measurement. The residue was dissolved in $100 \mu 1$ of ethylacetate and $2-5 \mu 1$ was injected into a gas chromatograph. 
The blood concentrations of AM were calculated from the GC peak area ratio of AM to AM- $d_{9}$. The distribution volume, the elimination rate constant and the clearance of AM were determined from the time course of blood concentration by applying the one-compartment model.

Gas Chromatography (GC) Conditions GC was carried out in a Shimadzu GC-14 A. A fused-silica capillary column (membrane thickness, $0.25 \mu \mathrm{m} ; 0.2 \mathrm{~mm}$ i.d. $\times 25 \mathrm{~m}$ ), a surface ionization type detector and a moving needle type solvent-cut injector were used. Helium gas linear flow, $74 \mathrm{~cm} / \mathrm{s}$; column temperature, $180^{\circ} \mathrm{C}$; injector temperature, $250^{\circ} \mathrm{C}$; detector temperature, $320^{\circ} \mathrm{C}$.

Pretreatment of Rats with IPN with or without AA Four days prior to the AM injection, $0.5 \mathrm{ml}$ of a saline solution of $\mathrm{PB}(70 \mathrm{mg} / \mathrm{kg} / \mathrm{d})$ was intraperitoneally injected for $3 \mathrm{~d}$ in order to induce the microsomal enzymes of liver, and at $24 \mathrm{~h}$ prior to the AM injection, $1 \mathrm{ml}$ of IPN in saline was injected (i.p.) at a dose $300 \mathrm{mg} / \mathrm{kg}$.

In the case of the combined administration of IPN with $\mathrm{AA}, 1 \mathrm{ml}$ of AA solution to 1.7 or 3 -fold molar amounts of IPN (AA, 491 or $884 \mathrm{mg} / \mathrm{kg}$ ) was given orally $1 \mathrm{~h}$ before the IPN administration. In the control group, $4 \mathrm{~d}$ prior to the AM injection, $P B(70 \mathrm{mg} / \mathrm{kg} / \mathrm{d})$ was administered as a pretreatment for $3 \mathrm{~d}$, as in the case of pretreatment with IPN.

Pretreatment of Rats with IP-Hy with or without AA As in the case of pretreatment with IPN, after the induction of microsomal enzymes, at $24 \mathrm{~h}$ prior to the AM injection, $1 \mathrm{ml}$ of IP-Hy sulfate $(120$ or $180 \mathrm{mg} / \mathrm{kg}$ ) in saline was injected (i.p.).

In the case of co-administration involving IP-Hy sulfate $(120 \mathrm{mg} / \mathrm{kg})$ with AA, $1 \mathrm{ml}$ of AA solution at 5 or 10 -fold molar amounts of IP-Hy (an equimolar mixture of AA and AA-Na, as AA 614 or $1228 \mathrm{mg} / \mathrm{kg}$ ) was given orally $1 \mathrm{~h}$ before the IP-Hy administration. In the case of co-administration of IP-Hy sulfate $(180 \mathrm{mg} / \mathrm{kg})$ with AA, AA consisting of 5-fold molar amounts of IP-Hy (an equimolar mixture of $\mathrm{AA}$ and $\mathrm{AA}-\mathrm{Na}$, as $\mathrm{AA} 812 \mathrm{mg} / \mathrm{kg}$ ) was administered (p.o.).

Determination of GOT and GPT Activities and Liver Histological Examination Blood collection and liver excision was performed $26 \mathrm{~h}$ after IPN or IP-Hy administration to the rats used for the measurement of AM clearance. Each blood sample was collected through a canule, as well as a sample for the measurement of AM clearance, and centrifuged. Determination of GOT and GPT activity was carried out with the plasma, using a commercially obtained kit (Transaminase C II-Test Wako). The excised liver was fixed with a $10 \%$ formalin neutral buffer solution ( $\mathrm{pH} 7.4)$, embedded with paraffin, cut into thin sections, and stained with Hematoxylineosin. The histological examination of these sections was done using microscopy.

\section{RESULTS AND DISCUSSION}

GOT and GPT activity after the administration of IPN or IP-Hy with or without AA is summarized in Table I.

The GOT and GPT activity after the administration of IPN or IP-Hy was significantly higher than that of the control group, indicating liver cell lesions. The mean
TABLE I. Effects of AA on GOT and GPT Activity after Administration of IPN or IP-Hy

\begin{tabular}{lrr}
\hline \hline \multicolumn{1}{c}{ Administration } & GOT (K.U.) & \multicolumn{1}{c}{ GPT (K.U.) } \\
\hline Control & $155.0 \pm 22.0$ & $34.0 \pm 5.1$ \\
IPN alone (300 mg/kg, i.p.) & $2537.7 \pm 299.4$ & $1801.9 \pm 200.4$ \\
with 1.7-fold molar AA & $2471.1 \pm 356.5$ & $1239.9 \pm 154.3$ \\
with 3-fold molar AA & $2938.5 \pm 91.0$ & $1597.0 \pm 148.7$ \\
IP-Hy alone (120 mg/kg, i.p.) & $843.8 \pm 177.3$ & $526.5 \pm 142.5$ \\
with 5-fold molar AA & $597.2 \pm 117.7$ & $337.8 \pm 84.0$ \\
with 10-fold molar AA & $639.3 \pm 91.7$ & $275.4 \pm 32.3$ \\
IP-Hy alone (180 mg/kg, i.p.) & $1198.5 \pm 281.1$ & $619.6 \pm 129.9$ \\
with 5-fold molar AA & $925.6 \pm 664.2$ & $456.0 \pm 316.4$ \\
\hline
\end{tabular}

Mean \pm S.E. $(n=3)$. K.U.: Karmen units.

values of GOT and GPT activity were found to have a tendency to be lower after the co-administration of IPN or IP-Hy with AA, compared with the single administration of either IPN or IP-Hy. However, the effects of AA could not be significantly evaluated by this variation of data. Hemolysis in vivo might be considered the cause for variation. It is well known that the existence of hemoglobin derived from hemolysis has an effect on GOT and GPT activity, so a measured value becomes larger than its real value. GOT and GPT activity is frequently used as an index of liver injury; however, the breakage of hepatic cells does not always reflect the level of hepatic function.

It has been reported that the metabolic clearance rates of AM correlate strongly with various measures of liver function in liver disorders. $\left.{ }^{8}\right)$ We previously reported ${ }^{6)}$ that the method based on the clearance of AM as an index for the evaluation of hepatic functions is an accurate method sensitively reflecting the metabolic function of liver. In the conventional evaluation of metabolic function, an AM breath test, ${ }^{8,9)}$ the radioactivity of $\left[{ }^{14} \mathrm{C}\right] \mathrm{CO}_{2}$ due to the demethylation of $\mathrm{AM}$ is monitored from exhaled breath after the administration of $\left[{ }^{14} \mathrm{C}\right] \mathrm{AM}$. On the other hand, the present method directly determines the eliminations rate from the time course of blood concentration of AM; therefore, the present method is considered to provide a more accurate evaluation of metabolic function. Since the determination of AM in the blood is carried out by isotope dilution analysis using $\mathrm{AM}-d_{9}$ as the internal standard, it is not necessary to use any radioactive isotope.

The blood concentration of AM after i.v. injection to rats after pretreatment with IPN $(300 \mathrm{mg} / \mathrm{kg})$ with or without AA is shown in Fig. 1.

The elimination of AM was more markedly delayed by pretreatment with IPN alone than that from the control, indicating a lowering of the level of metabolic function. Also, it was shown that the combined pretreatment of IPN with AA caused a more rapid elimination of AM, compared to the case of pretreatment with IPN alone. The kinetic parameters of AM, estimated from the time course of blood concentration by applying a one compartment model, are summarized in Table II.

The elimination rate constant $\left(k_{\mathrm{el}}\right)$ of AM noticeably increased, 2 times higher, following the combined pretreatments with $\mathrm{AA}$ in all cases of dosing with $\mathrm{AA}$, compared to the case of pretreatment with IPN alone. A significant increase in the total body clearance $(C L)$ of 


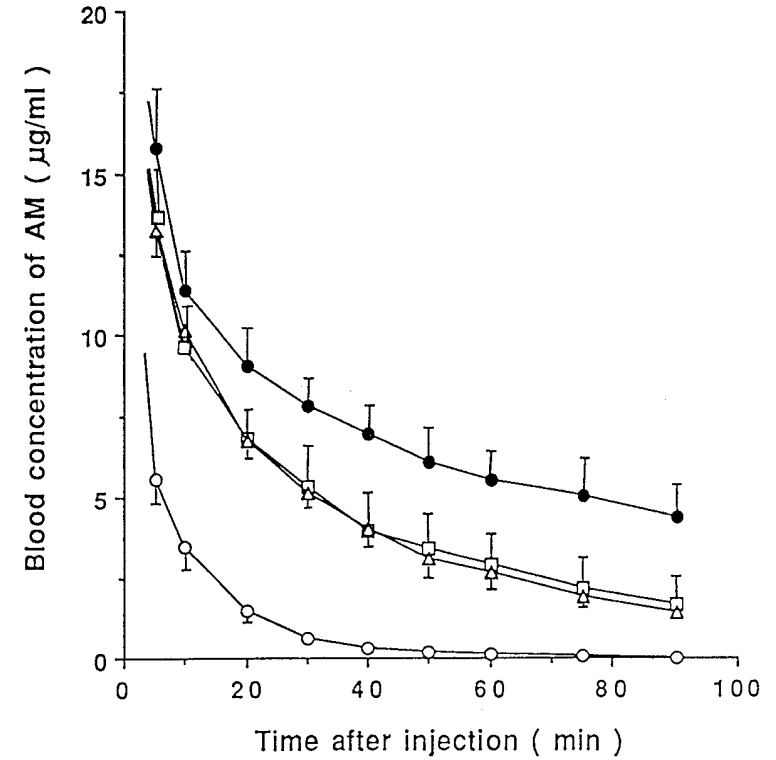

Fig. 1. Blood Concentration of AM after i.v. Injection to Rats after Pretreatment with IPN $(300 \mathrm{mg} / \mathrm{kg}$, i.p.) with or without AA

Each point represents the mean \pm S.E. of 3 rats. Key: - IPN alone; $\square$, with 1.7-fold molar AA; $\triangle$, with 3-fold molar AA; $O$, control.

TABLE II. Effects of AA on Kinetic Parameters of AM in Rats Pretreated with IPN $(300 \mathrm{mg} / \mathrm{kg}$, i.p.)

\begin{tabular}{lccc}
\hline \hline \multicolumn{1}{c}{ Administration } & $V_{\mathrm{d}}(\mathrm{l} / \mathrm{kg})$ & $k_{\mathrm{e} 1}\left(\mathrm{~h}^{-1}\right)$ & $C L(\mathrm{l} / \mathrm{kg} \cdot \mathrm{h})$ \\
\hline Control & $1.33 \pm 0.23$ & $5.00 \pm 0.10$ & $6.63 \pm 1.11$ \\
IPN alone & $0.83 \pm 0.09$ & $0.81 \pm 0.21$ & $0.66 \pm 0.13$ \\
with 1.7-fold molar AA & $0.87 \pm 0.11$ & $1.61 \pm 0.27$ & $1.46 \pm 0.40$ \\
with 3-fold molar AA & $0.77 \pm 0.01$ & $1.77 \pm 0.29$ & $1.36 \pm 0.21^{a)}$ \\
\hline
\end{tabular}

Mean \pm S.E. $(n=3)$, a) $p<0.05$, compared with IPN alone.

AM was also proved in the case of combined pretreatment with AA at 3-fold moles of AA. It could not be determined with the dose range of AA used in the experiment (up to 3-fold molar AA) whether or not AA affects the toxicity of IPN in a dose-dependent manner. Experiments with higher doses of AA were not performed because AA is difficult to dissolve in $1 \mathrm{ml}$ of water. We had previously confirmed that IPN does not influence the pharmacokinetics of IPN. ${ }^{5)}$ It seems unlikely, therefore, that pretreatment with AA reduced liver damage by promoting the renal excretion of IPN.

The blood concentration of AM after i.v. injection to rats following pretreatment with IP-Hy sulfate (120 and $180 \mathrm{mg} / \mathrm{kg}$ ) with or without AA is shown in Figs. 2, 3, and the kinetic parameters of AM estimated in the same manner as described above are summarized in Table III.

As in the case of pretreatment with IPN alone, the elimination of AM was significantly delayed by pretreatment with IP-Hy sulfate alone (120 and $180 \mathrm{mg} / \mathrm{kg}$ ) compared to that of the control, indicating a lowering of the level of metabolic function.

In our previous experiments with a microsomal system, ${ }^{5)}$ the intensity of the isopropyl radical generated by IP-Hy was twice as high as that of the isopropyl radical generated by equimolar IPN. In the present experiment, the severity of liver damage induced by IP-Hy pretreatment was

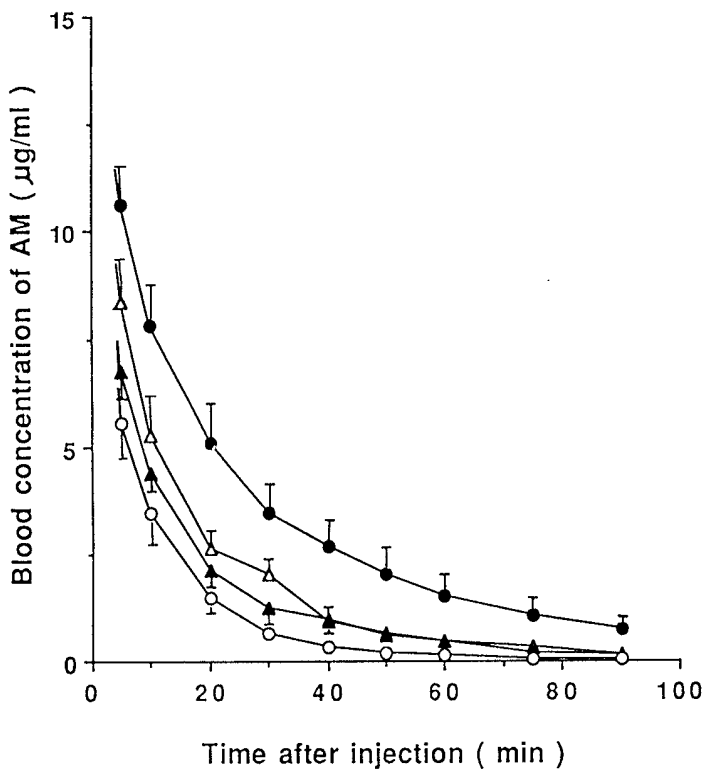

Fig. 2. Blood Concentration of AM after i.v. Injection to Rats after Pretreatment with IP-Hy Sulfate $(120 \mathrm{mg} / \mathrm{kg}$, i.p.) with or without AA

Each point represents the mean \pm S.E. of 3 rats. Key:, IP-Hy alone; $\triangle$, with 5-fold molar AA; $\boldsymbol{\Delta}$, with 10-fold molar AA; $\mathrm{O}$, control.

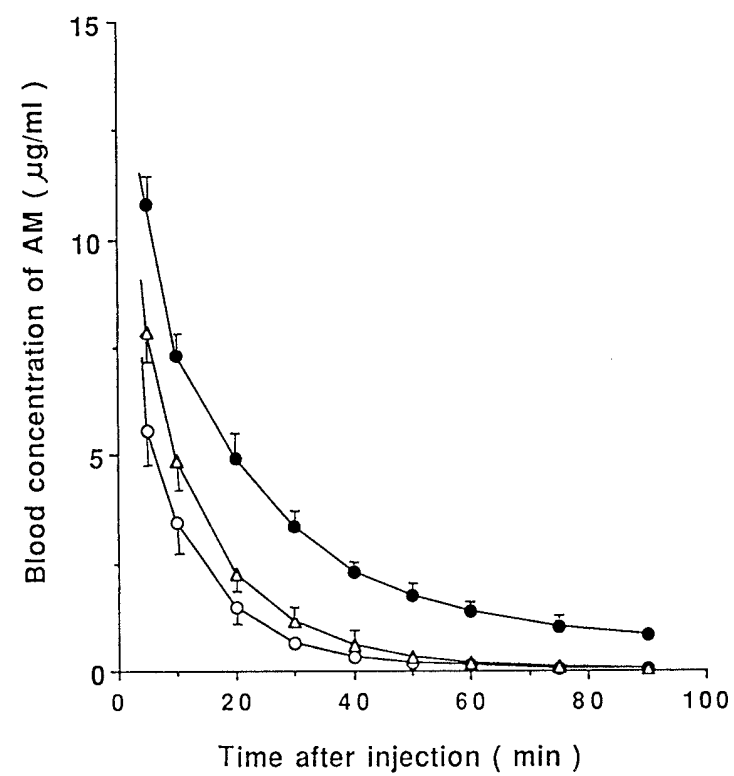

Fig. 3. Blood Concentration of AM after i.v. Injection to Rats after Pretreatment with IP-Hy Sulfate $(180 \mathrm{mg} / \mathrm{kg}$, i.p.) with or without AA

Each point represents the mean \pm S.E. of 3 rats. Key: - IP-Hy alone; $\triangle$, with 5-fold molar AA; O, control.

TABLE III. Effects of AA on Kinetic Parameters of AM in Rats Pretreated with IP-Hy

\begin{tabular}{clll}
\hline \hline Administration & $V_{\mathrm{d}}(\mathrm{l} / \mathrm{kg})$ & $k_{\mathrm{el}}\left(\mathrm{h}^{-1}\right)$ & \multicolumn{1}{c}{$C L(\mathrm{l} / \mathrm{kg} \cdot \mathrm{h})$} \\
\hline Control & $1.33 \pm 0.23$ & $5.00 \pm 0.10$ & $6.63 \pm 1.11$ \\
IP-Hy alone (120 mg/kg) & $0.92 \pm 0.05$ & $2.17 \pm 0.31$ & $2.00 \pm 0.30$ \\
with 5-fold molar AA & $1.25 \pm 0.29$ & $3.18 \pm 0.50$ & $3.69 \pm 0.50^{b)}$ \\
with 10-fold molar AA & $1.36 \pm 0.11$ & $3.38 \pm 0.12^{a)}$ & $4.59 \pm 0.36^{c)}$ \\
Ip-Hy alone (180 mg/kg) & $0.99 \pm 0.05$ & $2.09 \pm 0.20$ & $2.06 \pm 0.13$ \\
with 5-fold molar AA & $1.00 \pm 0.05$ & $4.43 \pm 0.28^{b)}$ & $4.44 \pm 0.52^{b)}$
\end{tabular}

Mean \pm S.E. $(n=3) . \quad$ a) $p<0.05, b) p<0.02, c) p<0.005$ compared with IP-Hy alone, respectively. 
determined to be lower than that induced by IPN based on biochemical and kinetic parameters. This is probably due to the fact that the IP-Hy doses were smaller than IPN doses (approximately $1 / 2 \mathrm{~mol}$ ) and that IP-Hy is metabolized and excreted more rapidly than IPN with lower tissue penetration. Nelson and his co-workers ${ }^{2)}$ studied the metabolism of IPN and IP-Hy labeled with tritium and ${ }^{14} \mathrm{C}$ in the methine position of the isopropyl group and they demonstrated that the metabolism and excretion of IP-Hy is more rapid than IPN.

Also, it was shown that the combined pretreatment with IP-Hy and AA, in all cases of dosing with AA, caused a more rapid elimination of AM, compared to the case of pretreatment with IP-Hy alone.

The $k_{\mathrm{el}}$ of $\mathrm{AM}$ showed a 1.5 times increase with the combined pretreatment with AA than in the case of pretreatment with IP-Hy sulfate alone $(120 \mathrm{mg} / \mathrm{kg})$, and an increase 2 times increase with the combined pretreatment with AA than in the case of pretreatment with IP-Hy sulfate alone $(180 \mathrm{mg} / \mathrm{kg})$. The significant increase in the $C L$ of AM was also observed in the case of combined pretreatment with AA in all cases of dosing with AA. Thus, the evaluation method for the metabolic function of liver using the $C L$ of AM as an index revealed that the $C L$ of AM is significantly increased by combined pretreatment with AA. It was confirmed that AA has a reducing effect on hepatic injury caused by IPN.

The microsomal enzyme induction with PB was carried out to produce a more distinct hepatic injury due to IPN or IP-Hy. Nelson et al. ${ }^{2)}$ reported that pretreatment with PB greatly potentiated the extent of necrosis due to IPN or IP-Hy, and the PB-pretreated rats, therefore, were used as a model for hepatic necrosis induced by IPN.

The effects of AA on hepatic injury caused by IPN were studied according to histological aspects. The sections of liver from rats administered IPN with or without AA are shown in Fig. 4.

Microscopic images of the liver tissue of the control showed that radially arranged rows of normal cells were oriented toward the central vein (Fig. 4A). In the specimen
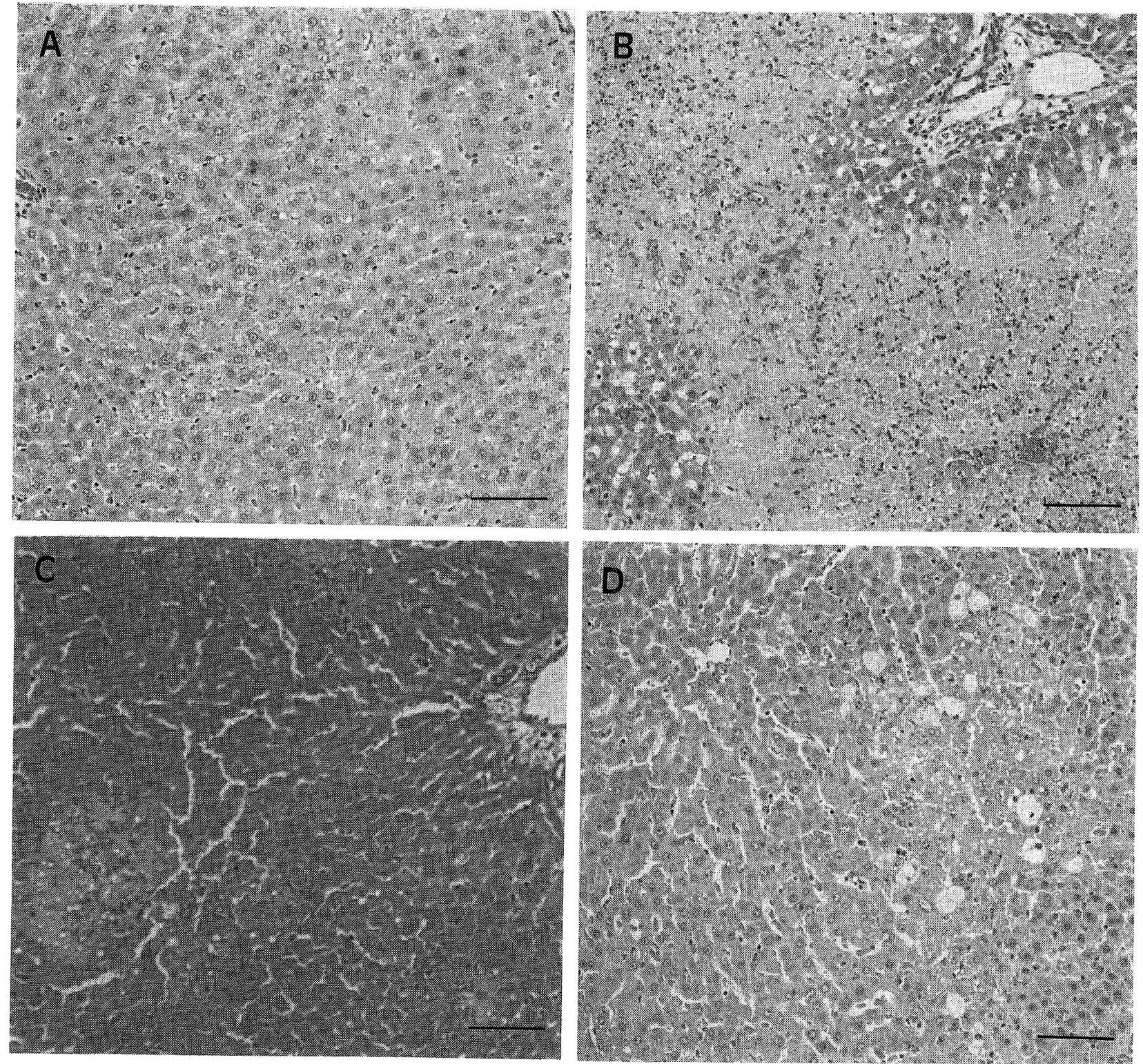

Fig. 4. Sections of Liver from Rats Administered IPN ( $300 \mathrm{mg} / \mathrm{kg}$, i.p.) with or without AA

Hematoxylin-eosin stain. Bar, $100 \mu \mathrm{m}$; A, control; B, IPN alone; C, with 1.7-fold molar AA; D, with 3-fold molar AA. 
obtained from the group which received an administration of IPN alone, massive cell necrosis was observed over a wide range, resulting in the development of detachment (Fig. 4B). The hepatic function was histologically estimated in an inhibited state. In the group administered 1.7-fold moles of AA, cell necrosis was also noted, but these cells were more sparsely distributed (Fig. 4C). As the dose of AA increased to 3-fold moles, the degree of cell necrosis became remarkably low both in quantitative and in qualitative aspects (Fig. 4D). Concerning the cell necrosis caused by IP-Hy, co-administration with AA also improved the cell necrosis to a great extent. These histological examinations of the rats' livers revealed that several rats seemed to have undergone the same type of changes in liver cells. However, these changes were not analyzed quantitatively.

These histological examinations also confirmed that AA has a marked effect on reducing liver injury by IPN, and these results of histological investigation agreed very closely with the results of evaluation of metabolic function using the $C L$ of AM.

In respect to the mechanism of development of hepatic insufficiency caused by drugs, it is known that the free radicals generated in the body may bind to unsaturated fatty acids, the constituent of phospholipids in the cell membrane, and act as the trigger of the chain reaction of lipid peroxidation, resulting in a lesion of the membrane and cell necrosis. ${ }^{10)}$ Development of hepatic injury due to IPN is also considered to be caused by a similar mechanism.

In our previous study, ${ }^{5)}$ free radical generation due to IPN and IP-Hy was confirmed by electron spin resonance spectroscopy and spin-trapping techniques using $\alpha$-(4pyridyl 1 -oxide)- $N$-tert-butylnitrone (4-POBN) as a spin trapping agent, and the 4-POBN-trapped radical species generated from IPN and IP-Hy were identified as isopropyl radical by the results of mass spectrometry by chemical ionization using isobuthane as a reagent gas.

Further, in a microsomal system, free radical generation was significantly inhibited by the addition of AA in a dose-dependent manner, and in in vivo experiments, the radical generation after i.p. administration of IPN with AA was significantly depressed in plasma and liver, compared with radical generation after the administration of IPN alone. Based on these results, it was suggested that AA may reduce the damage caused by IPN-induced hepatitis. ${ }^{5)}$
In the present study, metabolic function was evaluated using the clearance of AM as an index of hepatic function, and it was indicated that an improvement of the $C L$ of AM can be obtained from the combined administration of AA. In addition, it was confirmed that co-administration with AA caused a significant decrease in liver cell necrosis by these histological examinations. Thus, AA was demonstrated to be effective in reducing hepatic injury caused by IPN.

Acknowledgments We are very grateful to Dr. Noriko Kagawa and Mrs. Mayumi Umeno, School of Medical Sciences, The University of Tokushima, for histological examination and observation. This work was supported in part by a Grant-in-Aid for Scientific Research (05671899) from the Ministry of Education, Science and Culture, Japan.

\section{REFERENCES}

1) A. Kumar, P. K. Misra, R. Mehotra, Y. G. Govil, G. S. Rana, Am. Rev. Respir. Dis., 143, 1350 (1991); B. Toth, J. Cancer Res. Clin. Oncol., 97, 97 (1980); E. S. Fiala, Cancer, 40, 2436 (1977); J. R. Michell, U. P. Thorgersson, M. Black, J. A. Timblell, W. R. Snodgrass, W. Z. Potter, D. J. Jollow, H. R. Keiser, Clin. Pharmacol. Ther., 18, 70 (1975); J. R. Mitchell, D. J. Jollows, Gastroenterology, 68, 392 (1975).

2) S. D. Nelson, J. R. Mitchell, W. R. Snodgrass, J. A. Timbrell, $J$. Pharmacol. Exp. Ther., 206, 574 (1978).

3) Y. Matsuki, Y. Katakuse, H. Matsuura, H. Kiwada, T. Goromaru, Chem. Pharm. Bull., 39, 445 (1991); Y. Matsuki, T. Goromaru, H. Matsuura, Yakugaku Zasshi, 103, 1219 (1983).

4) Y. Matsuki, M. Akazawa, K. Tsutiya, H. Sakurai, K. Kiwada, T. Goromaru, Yakugaku Zasshi, 111, 600 (1991).

5) Y. Matsuki, Y. Hongu, Y. Noda, K, Kiwada, H. Sakurai, T. Goromaru, Yakugaku Zasshi, 112, 926 (1992).

6) T. Goromaru, H. Maeda, Y. Matsuki, H. Kiwada, Radioisotopes, 42, 511 (1993).

7) T. Goromaru, H. Ikejiri, K. Hashimoto, Y. Matsuki, Yakugaku Zasshi, 111, 612 (1991).

8) G. W. Hepner, E. S. Vesell, Clin. Pharmacol. Ther., 20, 654 (1976); idem, Ann. Intern. Med., 83, 632 (1975).

9) V. C. Ramin, J. Bircher, Eur. J. Clin. Pharmacol., 38, 87 (1990); T. Miotti, J, Bircher, R. Preisig, Digestion, 39, 241 (1988); A. L. Baker, P. S. Krager, A. N. Kotake, D. A. Schoeller, Hepatology, 7, 464 (1987); E. Dicker, A. I. Cederbaum, J. Pharmacol. Exp. Ther., 227, 687 (1983).

10) G. M. Rosen, W. V. Singletary, E. J. Rauckman, G. Paul, Biochem. Pharmarol, 32, 2053 (1983); H. D. Groot, T. Noll, Hepatology, 3, 601 (1983); B. H. Bielski, R. L. Arudi, M. W. Sutherland, J. Biol. Chem., 258, 4759 (1983). 